\title{
ESTUDIO DEL EFECTO MODIFICADOR DEL Na PROVENIENTE DEL NaCI SOBRE LA MORFOLOGÍA DEL Si EN UNA ALEACIÓN Al-Si HIPOEUTÉCTICA
}

\section{STUDY OF MODIFIER EFFECT OF SODIUM ARISING FROM NaCI UPON THE MORPHOLOGY OF SILICON IN AN HYPOEUTECTIC Al-Si ALLOY}

\author{
Enrique J. Martínez D. ${ }^{1} \quad$ Rubin Ortega de la Rosa $^{1} \quad$ Gerardo Torres C. $^{2}$ \\ Recibido 6 de marzo de 2006, aceptado el 7 de agosto de 2006 \\ Received: March 6, 2006 Accepted: August 7, 2006
}

\begin{abstract}
RESUMEN
Este trabajo comprende el estudio del efecto del sodio proveniente del $\mathrm{NaCl}$ (halita), sobre la morfología del Si eutéctico en una aleación Al-Si hipoeutéctica vaciada. La fusión se llevó a cabo en dos hornos, el primero a combustión de gas LP con crisol de grafito para incrementar el contenido de Si en la aleación, de 0.13 a $5 \%$ en peso utilizando silicio metálico con una pureza de $99.35 \%$, y el segundo de resistencias eléctricas con el mismo tipo de crisol, para controlar la temperatura a $750{ }^{\circ} \mathrm{C}$. El material fundido fue vaciado en moldes de arena shell, diseñados para evitar impurezas e interacciones en las zonas de estudio. Para añadir la sal al baño metálico, se usaron dos técnicas: una con insuflación mediante gas argón y la otra por gravedad, en ambos casos se manejaron tres cantidades de sal, $0.5,1.5$ y $2.5 \%$ en peso respectivamente, pulverizada y precalentada a $150{ }^{\circ} \mathrm{C}$ durante $60 \mathrm{~min}$. Mediante análisis químico se determinó el contenido de $\mathrm{NaCl}$ en la sal siendo de $99.5 \%$ en peso, con este dato se calculó la cantidad teórica de sal necesaria. Las muestras obtenidas fueron analizadas metalográficamente, evidenciando la modificación morfológica del Si eutéctico en la escala \#2 a \#4 de acuerdo a la literatura, presentando los mejores resultados las muestras con 1.5 y $2.5 \%$ de sal agregada por gravedad. Finalmente, mediante microscopia electrónica de barrido se caracterizaron los precipitados presentes en las muestras.
\end{abstract}

Palabras clave: Na, modificación, Al-Si, $\mathrm{NaCl}$, solidificación.

\begin{abstract}
This work, comprehends a study about the effect of sodium obtained from $\mathrm{NaCl}$ (halita) upon the morphology of eutectic silicon in a cast hypoeutectic Al-Si alloy. The melting was carried out in two furnaces, the first was done using LP gas combustion with a graphite crucible for increasing the content of Si in the alloy from 0.13 to $5 \%$ wt, using metallic silicon with a purity of $99.35 \% \mathrm{wt}$. The second melting was done using electric resistances with the same kind of crucible to control the temperature at $750^{\circ} \mathrm{C}$. The melting material was cast into molds of shell sand, to avoid sludge and interaction in the study areas. To add the salt to the metallic bath, two techniques were used; one with injection by means of argon gas and the other one by gravity. In both cases three quantities of salt were used, 0.5, 1.5 and 2.5\% in weight respectively, powdered and preheated at $150^{\circ} \mathrm{C}$ during $60 \mathrm{~min}$. The content of $\mathrm{NaCl}$ in the salt, $95 \%$ wt was determined by chemical analysis. The theoretical quantity of necessary salt was calculated with this data. The obtained samples were analyzed metallographically, evidencing the modification of the eutectic Si in the scale \#2 at \#4, in agreement with the literature, presenting the best results the samples with $1.5 \& 2.5 \%$ wt of salt added by gravity. Finally, by means of scanning electron microscopy, the precipitate present was characterized in the samples.
\end{abstract}

Keywords: Na, modification, Al-Si, $\mathrm{NaCl}$, solidification.

\section{INTRODUCCIÓN}

Las aleaciones de aluminio vaciado constituyen en términos de tonelaje el segundo grupo más grande de consumo de metales, después de las aleaciones de fierro vaciado [1] y en particular las tipo Al-Si son ampliamente utilizadas en la industria automotriz, estructuras aerospaciales y aplicaciones militares, debido

\footnotetext{
1 Instituto Tecnológico de Zacatecas, México, Carr. Panamericana entronque a Guad. S/N, e-mail enriquemartinez_1999@yahoo.com

2 Ciateq, El Marqués Querétaro, México.
} 
a sus buenas propiedades mecánicas, bajo peso, buena soldabilidad, buena conductividad térmica, relativamente alta resistencia a alta temperatura, excelente resistencia a la corrosión, así como excelente fluidez al vaciado [2]. Debido a la evolución de las microestructuras durante el proceso de solidificación, la microestructura no solamente determina las propiedades mecánicas, sino que también define los límites del diseño de vaciado, la colabilidad de la aleación y la susceptibilidad a la formación de defectos [3]. La forma del Si eutéctico juega un papel muy importante en las propiedades mecánicas de las aleaciones Al-Si, particularmente en su resistencia a la elongación, incrementando su ductilidad mediante el refinamiento estructural del silicio eutéctico cuya fase es inherentemente frágil; sin embargo, su morfología se modifica de placas aciculares gruesas a una estructura fibrosa (similar a un alga marina) o laminar fina, mediante la adición de pequeñas cantidades de los elementos de la familia IA, IIA y tierras raras de la tabla periódica, o bien con velocidades de enfriamiento altas $[4,5]$. El estroncio, sodio y antimonio son los tres elementos modificadores más utilizados comercialmente para tratar las aleaciones fundidas de Al-Si [4], K. Nogita y colaboradores [6] han reportado la modificación del eutéctico Al-Si con $\mathrm{Ba}, \mathrm{Ca}, \mathrm{Y}$ y $\mathrm{Yd}$, proponiendo sus mecanismos de solidificación. Desafortunadamente estudios recientes han demostrado que la modificación está asociada con efectos negativos tales como porosidad, agrietamiento y pobre acabado superficial $[4,7]$. A.K. Dahle y colaboradores [8] reportaron que el Sr es más potente su efecto modificador en el cambio morfológico del Si eutéctico que el Sb. También se ha reportado el uso de la combinación de elementos modificantes, sin embargo, se encontró que el $\mathrm{Na}$ y el $\mathrm{Sr}$ reaccionan con el $\mathrm{Sb}$ formando una serie de precipitados en el material fundido [4]. L. Lu y colaboradores [9] reportan en su trabajo que el efecto de la adición combinada del $\mathrm{Na}$ y $\mathrm{Sr}$ es prácticamente el mismo que el del $\mathrm{Na}$ al inicio, es decir, el cambio morfológico obtenido en ambos casos ( $\mathrm{Sr}-\mathrm{Na}$ y Na) fue el mismo; sin embargo, ellos encontraron que después de un tiempo de mantenimiento a $730{ }^{\circ} \mathrm{C}$ hay una interacción negativa entre estos dos modificadores, lo cual, según ellos, se debe a una pérdida por evaporación de estos elementos; así tenemos que, de todos los elementos modificadores, el sodio es el más poderoso, produciendo estructuras modificadas más uniformes a más bajas concentraciones que las que se producen con los de más; sin embargo, debido a que es un elemento muy reactivo, se ha limitado su uso en la industria debido a que se ha reportado que esta fuerte reacción origina absorción de hidrógeno de atmósfera. L. Bäckerud y colaboradores [5] reportan que después de dos fundiciones la acción modificadora del sodio prácticamente desaparece, lo cual no ha sido observado respecto al estroncio. El propósito de este trabajo es estudiar el efecto del Na proveniente de la sal común tipo gema sobre la morfología del silicio eutéctico en una aleación aluminio-silicio hipoeutéctica, buscando proponer una fuente diferente de elemento modificador, ya que en la actualidad solo se ha reportado la modificación del Si eutéctico usando elementos puros $o$ aleaciones maestras entre estos elementos y el aluminio y en muy pocas ocasiones fundentes como fluoruro de $\mathrm{Na}[9]$.

\section{EXPERIMENTACIÓN}

\section{Estudio del NaCl utilizado}

La sal utilizada en este trabajo fue obtenida de yacimientos minerales de la región de Villa de Cos Zacatecas, México, la cual se pulverizó manualmente en un mortero de laboratorio, obteniéndose asimismo su análisis granulométrico, se determinó su composición mediante análisis químico por vía húmeda (volumetría) y difracción de rayos X. Finalmente se realizó un análisis de determinación de humedad, tomando una muestra representativa de la sal mediante el método de cuarteos cuyo peso fue de aproximadamente $20 \mathrm{gr}$, tanto pulverizada como en grano, para posteriormente ser calentadas las muestras de sal en una mufla (horno de resistencias eléctricas) a $150{ }^{\circ} \mathrm{C}$ durante 60 minutos y por simple diferencia de pesos se determinó la cantidad de humedad desprendida.

\section{Obtención de muestras para estudio}

La aleación base para el presente estudio fue obtenida a partir de chatarra de aluminio de cancelería. La fundición se llevó acabo en dos etapas, en la primera se utilizó un horno con combustión de gas LP y aire insuflado con crisol de grafito de $30 \mathrm{~kg}$, en donde se incrementó el contenido de Si de 0.134 a $5 \%$ en peso, añadiendo al baño fundido Si metálico con una pureza de $99.35 \%$ en peso, para asegurar la disolución del Si se mantuvo la aleación líquida a $750{ }^{\circ} \mathrm{C}$ durante 30 minutos; en la segunda etapa se utilizó un horno eléctrico marca Carbolite modelo GPC 12/200 con pequeños crisoles de grafito de $3 \mathrm{~kg}$ únicamente para mantener la temperatura a $750{ }^{\circ} \mathrm{C}$. Tanto la aleación de chatarra fundida como la aleación base Al-SI fueron analizadas mediante espectrometría de emisión por chispa. En base a estudios previos [10], la sal fue añadida a la aleación líquida mediante dos técnicas, 1) insuflada con gas inerte (argón) y 2) por gravedad, con la finalidad de asegurar la mejor interacción entre los granos sólidos de $\mathrm{NaCl}$ y el baño metálico; en ambos 
casos se manejaron tres cantidades de sal, $0.5,1.5$ y $2.5 \%$ en peso respectivamente, pulverizada y precalentada a $150{ }^{\circ} \mathrm{C}$ durante 60 minutos.

La aleación fundida y tratada con $\mathrm{NaCl}$ fue vaciada en moldes de arena obtenidos mediante el proceso shell (figura 1a) cuyo diseño fue de tal manera de evitar impurezas e interacciones en las zonas de estudio de donde se obtuvieron las muestras para su análisis. La tabla 1 muestra la nomenclatura utilizada.

\section{Análisis microestructural}

Una vez cortadas las muestras (figura 1b), estas fueron preparadas metalográficamente mediante las técnicas convencionales y observadas en un microscopio óptico metalográfico de platina invertida marca Iroscope; asimismo se hicieron mediciones de espaciamiento dendrítico secundario promedio (DAS) a cada muestra mediante un analizador de imágenes marca Sigma Scan Pro 5. Para analizar químicamente los precipitados presentes se utilizó un microscopio electrónico de barrido (MEB) marca FEI modelo Quanta 200 con EDX Oxford Instruments.
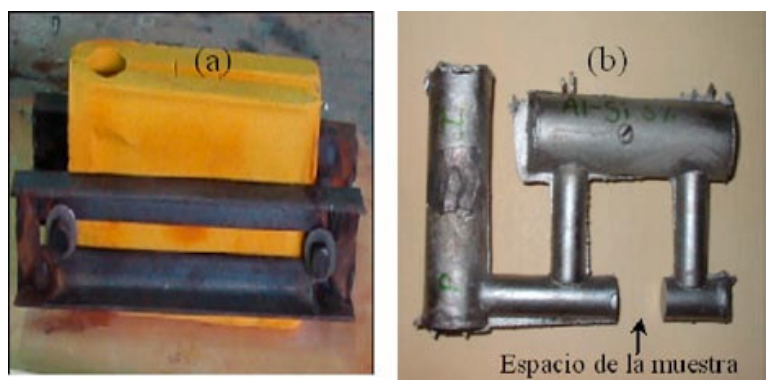

Figura 1. Fotografías mostrando (a) Molde de arena shell y (b) Sección de donde se cortaron las muestras.

Tabla 1. Nomenclatura utilizada en las muestras.

\begin{tabular}{|c|c|c|c|}
\hline Modo de aplicación & \multicolumn{3}{|c|}{$\%$ en peso de sal } \\
\hline & 0.5 & 1.5 & 2.5 \\
\hline Inyección con argón & A5 & A15 & A25 \\
\hline Por gravedad & G5 & G15 & G25 \\
\hline
\end{tabular}

\section{RESULTADOS Y DISCUSIÓN}

\section{Estudio del NaCl utilizado}

La figura 2 muestra los resultados obtenidos del análisis granulométrico de la sal utilizada, tanto en grano ( $\sin$ moler) como pulverizada.

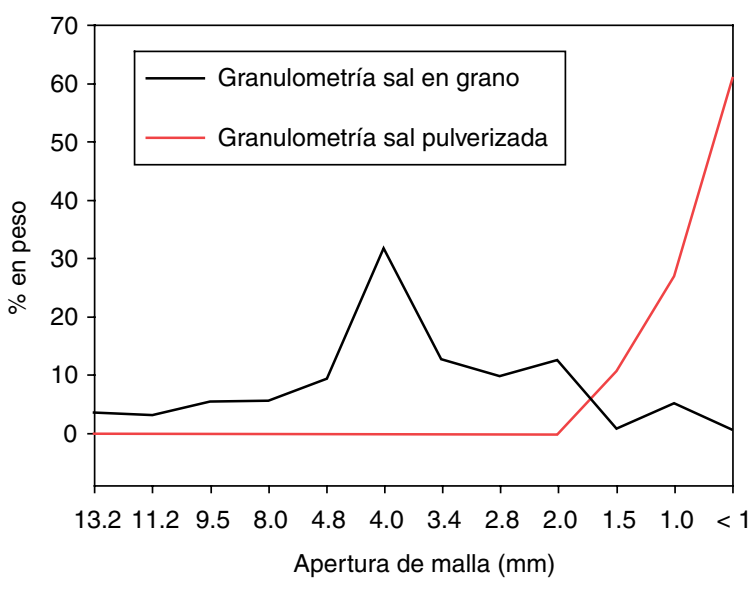

Figura 2. Gráfica de la distribución del tamaño de las partículas de sal.

La gráfica de la figura 2 muestra que el mayor porcentaje del tamaño de grano para la sal sin moler se encuentra entre 3 y $5 \mathrm{~mm}$, mientras que para la sal pulverizada se encuentra a un tamaño menor a $1 \mathrm{~mm}$, lo indica que la sal pulverizada tendrá una mayor área superficial, facilitando el desprendimiento de humedad, esto explica los resultados del análisis de humedad, $24.1 \%$ en peso para la sal pulverizada y $3.25 \%$ en peso para la sal sin moler.

La tabla 2 muestra los resultados obtenidos del análisis químico de la sal utilizada. La figura 3 muestra los resultados obtenidos en el análisis de difracción de rayos $\mathrm{X}$.

Tabla 2. Reporte del análisis químico de la sal.

\begin{tabular}{|c|c|c|c|c|}
\hline $\mathrm{NaCl}$ & Ion sulfato & Ion $\mathrm{Mg}$ & Ion $\mathrm{Ca}$ & Insolubles \\
\hline 99.547 & 0.035 & 0.218 & 0.067 & 0.073 \\
\hline
\end{tabular}

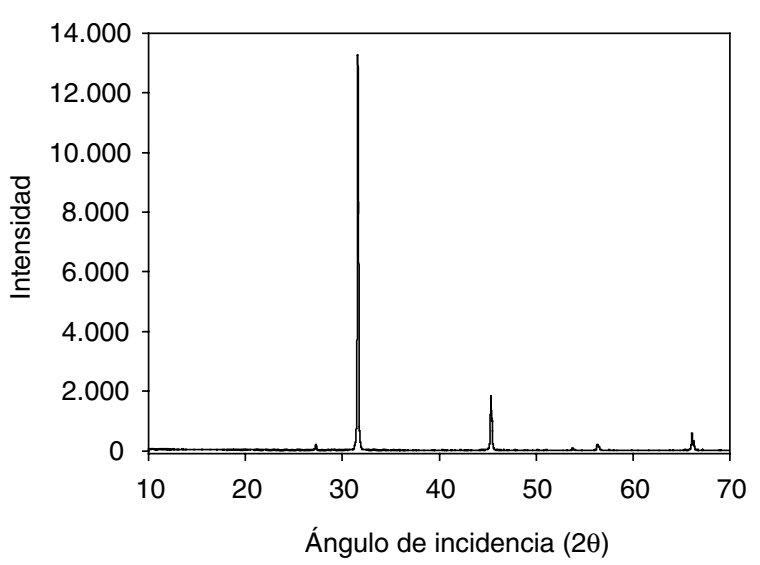

Figura 3. Difractograma correspondiente a la sal utilizada en este trabajo. 
Tomando en cuenta la pureza del $\mathrm{NaCl}$ obtenida de la tabla 2, se calculó la cantidad teórica necesaria de sal para desgasificar la aleación considerando la ecuación (1) propuesta por John E. Gruzleski y colaboradores [4] y suponiendo que todo el cloro del $\mathrm{NaCl}$ reacciona con el hidrógeno del baño metálico, lo cual dio como resultado $0.33 \%$ en peso de sal para eliminar la totalidad del hidrógeno en la aleación líquida. En base a este cálculo se establecieron los parámetros experimentales de la adición de sal.

$$
\log _{10} S=\frac{-2550}{T}+2,62
$$

\section{Obtención de muestras para estudio}

Las tablas 3 y 4 muestran la composición química de la chatarra de aluminio y de la aleación base Al-Si respectivamente, obtenidas mediante espectrometría de emisión por chispa.

Tabla 3. Composición química (\% en peso) de la chatarra fundida.

\begin{tabular}{|c|c|c|c|c|c|c|c|}
\hline $\mathrm{Si}$ & $\mathrm{Fe}$ & $\mathrm{Cu}$ & $\mathrm{Mg}$ & $\mathrm{Zn}$ & $\mathrm{Mn}$ & $\mathrm{Sr}$ & $\mathrm{Ti}$ \\
\hline 0.134 & 0.416 & 0.013 & 0.005 & 0.006 & 0.015 & 0.0006 & 0.005 \\
\hline
\end{tabular}

Tabla 4. Composición química (\% en peso) de la aleación base Al-Si.

\begin{tabular}{|c|c|c|c|c|c|c|c|}
\hline $\mathrm{Si}$ & $\mathrm{Fe}$ & $\mathrm{Cu}$ & $\mathrm{Mg}$ & $\mathrm{Zn}$ & $\mathrm{Mn}$ & $\mathrm{Sr}$ & $\mathrm{Ti}$ \\
\hline 5.12 & 0.519 & 0.044 & 0.243 & 0.014 & 0.010 & 0.0009 & 0.012 \\
\hline
\end{tabular}

De las tablas 3 y 4 se puede observar que el aumento de silicio fue de acuerdo a lo esperado ( $5 \%$ en peso), asimismo se puede apreciar que hubo un incremento considerable de $\mathrm{Mg}$ en la aleación, esto debido a la interacción de la sal con el baño metálico. Otro aspecto interesante es que el estroncio permaneció prácticamente constante ya que se incrementó tan solo 3 ppm, lo cual, de acuerdo a la literatura $[4,11]$, no tiene efecto sobre la modificación del silicio eutéctico, las otras variaciones se debieron a la presencia de impurezas en el baño metálico.

\section{Análisis microestructural}

La figura 4 presenta las fotomicrografías de las muestras correspondientes a la chatarra fundida y aleación base Al-Si. En la figura 4b se puede apreciar que tiene mayor cantidad de silicio eutéctico con morfología acicular en la escala \#1 según lo reportado en la literatura respecto al rango total de microestructuras observadas en una aleación de aluminio hipoeutéctica modificada [4, 11] debido al incremento de su contenido en la aleación AlSi. Otra diferencia interesante que se puede apreciar en esta figura es en el tamaño de grano, siendo más grande en la muestra de chatarra fundida cuyo espaciamiento dendrítico secundario promedio (DAS) fue de 129.49 $\mu \mathrm{m}$, lo cual es debido a que contiene muy poco silicio y como no se utilizó refinador de grano, no presentó muchos sitios favorables para la nucleación; en contraste, en la muestra de la aleación base Al-Si se observó un grano un poco más fino con un DAS de 127.7 micras, fenómeno que se debió al efecto del silicio en la aleación, el cual incrementó la fase eutéctica de este elemento, ocasionando una disminución en el tamaño de grano dendrítico de la fase primaria $\alpha$-Al a diferencia de la muestra de chatarra fundida cuyas dendritas crecieron libremente, esto significa que la nucleación y crecimiento del eutéctico de silicio en este caso se dan en las puntas de las dendritas de $\alpha$-Al, lo que está en acuerdo a lo reportado por A. K. Dahle y colaboradores [12].

La figura 5 muestra las fotomicrografías de las muestras G5, G15 y G25. Comparando estas fotomicrografías con las de la figura 4 se observa un tamaño de grano más fino, debido al efecto refinador de los granos sólidos del $\mathrm{NaCl}$, los cuales actúan como sitios de nucleación [13]. Analizando la figura 5, la muestra G5 presentó una leve modificación de la morfología del silicio eutéctico, que de acuerdo al rango total de microestructuras reportadas en la literatura $[4,11]$ corresponde a una estructura de laminar a submodificada (\#2 a \#3), con un tamaño de grano pequeño (DAS de $76.74 \mu \mathrm{m}$ ), mientras que la muestra G15 presentó una estructura submodificada (\#3 a \#4) de acuerdo a la escala de modificación $[4,11]$, con un DAS promedio de $84.0 \mu \mathrm{m}$, debido a la mayor cantidad de sal utilizada. En la muestra G25 se observó una morfología del Si eutéctico de submodificada a bien modificada de acuerdo a la literatura $[4,11]$, con un espaciado dendrítico secundario de $64.6 \mu \mathrm{m}$.

Considerando la cantidad teórica de Na contenida en la sal y agregada al baño metálico, esto es: 1956, 5868 y 9780 ppm de sodio al agregar 0.5, 1.5 y $2.5 \%$ en peso de $\mathrm{NaCl}$, respectivamente, estas cantidades están muy por arriba de lo reportado en la literatura (de 100 a $200 \mathrm{ppm}$ ) para tener una buena modificación de la morfología del Si eutéctico [4, 11]. No se obtuvieron resultados excelentes de la modificación morfológica del Si eutéctico a pesar de tener teóricamente un exceso de $\mathrm{Na}$ en el sistema, debido a la limitada disociación del $\mathrm{NaCl}$ en el baño metálico, lo cual se comprobó en forma cualitativa mediante experimentos de la interacción entre una briqueta de $\mathrm{NaCl}$ sólido y una gota líquida de 
aleación de Al-Si, obteniéndose un ángulo de contacto grande $\left(75^{\circ}\right)$, y de acuerdo a la teoría de nucleación [13] implica que hay mojabilidad limitada de la sal en la aleación Al-Si, lo que debería ser estudiado con más profundidad.
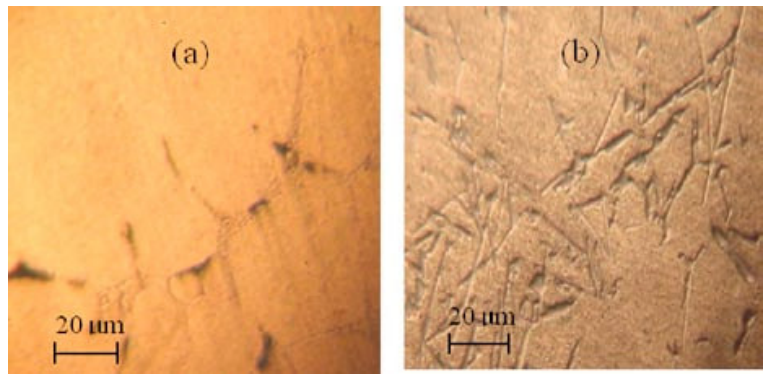

Figura 4. Fotomicrografía mostrando las fases de $\mathrm{Si}$ eutéctico y las dendritas de $\alpha$-Al primario, a). En la chatarra fundida y b). En la aleación base Al-Si.

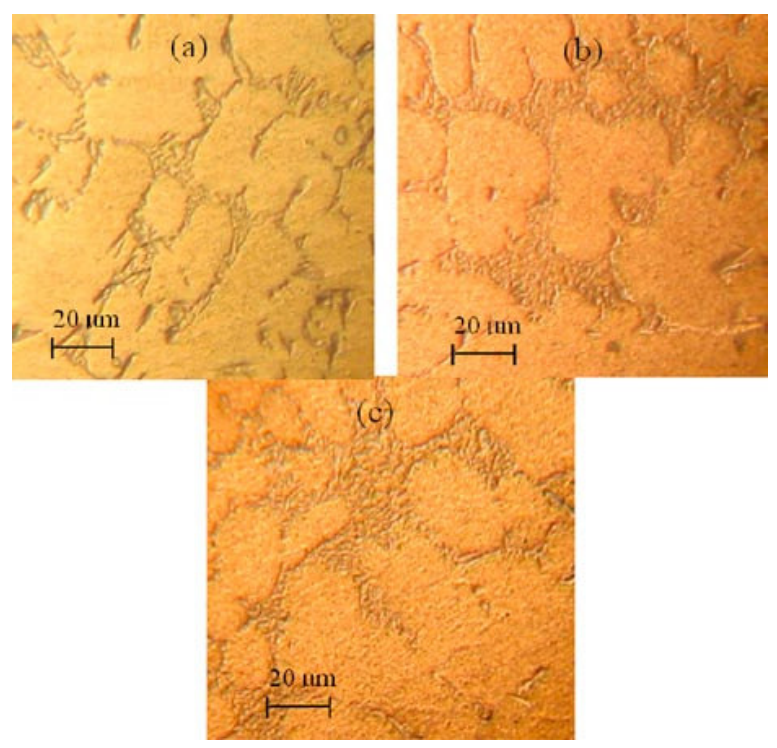

Figura 5. Fotomicrografías mostrando las estructuras modificadas del Si eutéctico, de las muestras a) G5, b) G15 y c) G25.

Las muestras A5, A15 y A25 no exhibieron ninguna modificación en la morfología del silicio eutéctico, esto se debió a que el argón presentó un efecto desfavorable a la modificación del silicio eutéctico, debido a que el $\mathrm{Na}$ es muy volátil [5] y fue arrastrado por el gas inerte, lo cual de alguna manera está en acuerdo con la teoría propuesta por L. Lu y colaboradores [9], ya que ellos proponen que el desvanecimiento de los elementos modificadores se debe a su evaporación, sin embargo este efecto aún no es claro.
Los resultados obtenidos del análisis de microscopia electrónica de barrido indicaron la presencia de cinco fases muy parecidas a las reportadas por F. H. Samuel y colaboradores [14], así como por E. J. Martínez D. y colaboradores [15], cuya diferencia fue en su relación atómica debido a la diferencia en composición química, sin embargo, en cuanto a morfología y distribución fue prácticamente la misma.

La tabla 5 reporta el análisis de los espectros señalados en la figura 6 , obtenidos del análisis químico puntual mediante EDX con microscopia electrónica de barrido (MEB) de la muestra G5.

Tabla 5. Reporte del análisis químico elemental de los espectros señalados en la figura 6 , obtenidos del MEB.

\begin{tabular}{|c|c|c|c|c|c|c|}
\hline \multirow{2}{*}{ Espectro } & \multicolumn{5}{|c|}{ Elemento } & \multirow{2}{*}{ Total } \\
\cline { 2 - 7 } & $\mathrm{Mg}$ & $\mathrm{Al}$ & $\mathrm{Si}$ & $\mathrm{Fe}$ & $\mathrm{Cu}$ & \\
\hline 1 & & 61.9866 & 7.8966 & & 30.1166 & 100 \\
\hline 2 & 6.5 & 65.05 & 6.62 & 4.22 & 17.62 & 100 \\
\hline 3 & 8.54 & 66.48 & 7.4 & & 17.58 & 100 \\
\hline 4 & & 81.3066 & 9.5166 & & 9.1666 & 100 \\
\hline
\end{tabular}

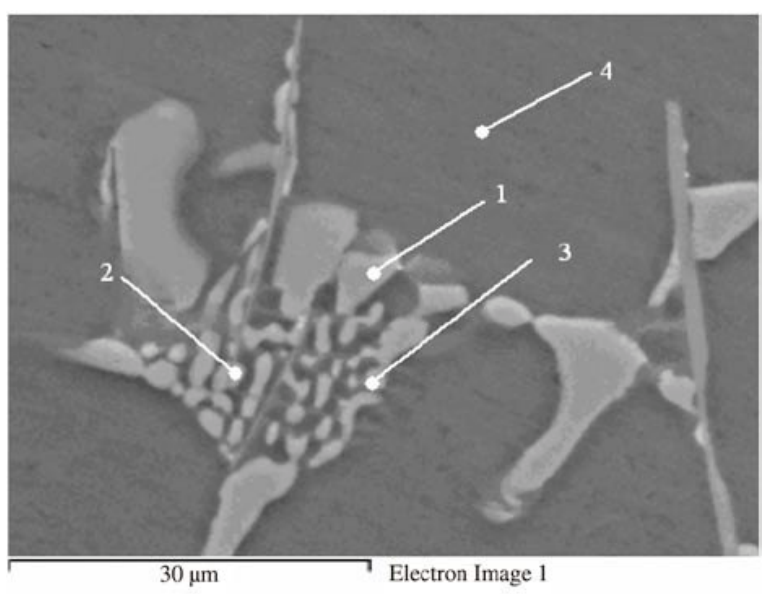

Figura 6. Fotomicrografía de la muestra G5, obtenida mediante EDX, mostrando fases ricas en $\mathrm{Fe}$, $\mathrm{Cu}$ y $\mathrm{Mg}$.

La figura 7 muestra las fotomicrografías obtenidas del mapeo químico de la muestra G5, usando EDX en el MEB, en la cual se puede apreciar la distribución de los elementos en las fases estudiadas, donde las zonas brillantes indican la riqueza del elemento en cuestión. Durante el análisis por microscopia óptica y electrónica se observaron cinco fases en todas las muestras. Las fases ricas en $\mathrm{Cu}$ se presentaron con morfología tipo "Blocky" o masiva, favoreciendo su formación hacia las zonas interdendríticas; se pudo apreciar que esta fase se presentó 
en más abundancia en las muestras G15 y G25, debido a que se favorece en presencia de elementos modificantes, lo cual está de acuerdo por lo reportado por Samuel [14] y Martínez [15]; la fase rica en cobre con morfología de eutéctico fino no se observó en estas muestras debido a su bajo contenido de este elemento en la aleación.

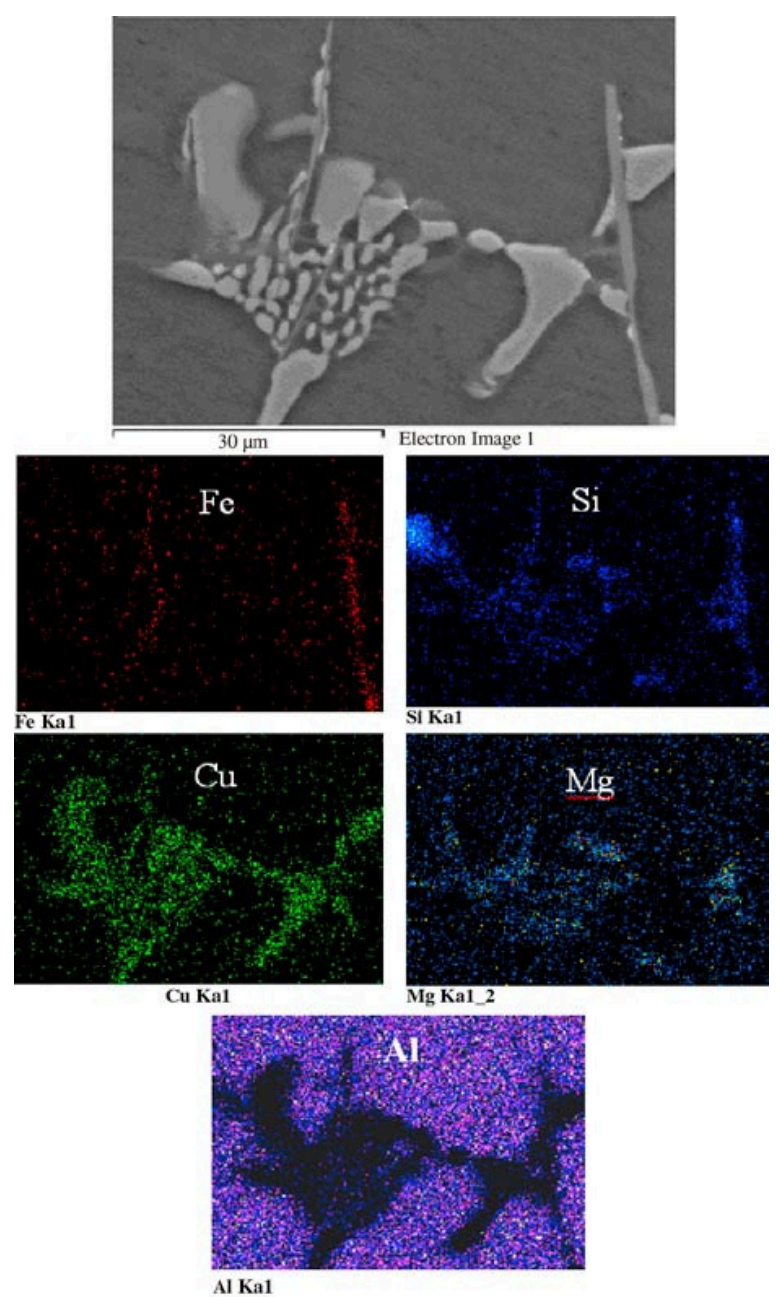

Figura 7. Resultado del mapeo químico obtenido de la muestra G5, mediante EDX en MEB. La figura más grande corresponde a la zona de estudio, mientras que las demás corresponden al mapa elemental, donde la brillantez indica la riqueza del elemento señalado.

También se observaron fases en forma de agujas, las cuales corresponden a la fase rica en hierro $\beta-\mathrm{Al}_{5} \mathrm{FeSi}[14,15]$. De acuerdo a la literatura existe otra fase rica en Fe en forma de escritura china, $\alpha-\mathrm{Al}_{15}(\mathrm{Fe}, \mathrm{Mn})_{3} \mathrm{Si}_{2}$; sin embargo, en este estudio no se observaron, debido al bajo contenido de $\mathrm{Mn}$, ya que este favorece la formación de este tipo de fase
[14]. Las fases ricas en silicio corresponden al eutéctico principal, el cual se obtuvo con morfología acicular en ausencia de modificación y en forma de alga marina [5] o estructura fibrosa cuando sufrió modificación con el $\mathrm{Na}$ proveniente del $\mathrm{NaCl}$. Finalmente, se pudo observar la presencia de la fase rica en magnesio $\mathrm{Al}_{5} \mathrm{Mg}_{8} \mathrm{Cu}_{2} \mathrm{Si}_{6}$, la cual está asociada a las fases ricas en $\mathrm{Cu}$ con morfología masiva y las agujas de fases ricas Fe, lo cual corresponde a la reacción de precipitación multifásica $(\ell \rightarrow \alpha \mathrm{Al}+$ $\mathrm{Al}_{2} \mathrm{Cu}+\mathrm{Si}+\mathrm{Al}_{5} \mathrm{Mg}_{8} \mathrm{Cu}_{2} \mathrm{Si}_{6}$ ) que tiene lugar al final de la solidificación, esto fue reportado por E. J. Martínez y colaboradores [16].

\section{CONCLUSIONES}

- De acuerdo a los resultados obtenidos, existe interacción entre el $\mathrm{Na}$ proveniente del $\mathrm{NaCl}$, debido a que se obtuvo modificación del silicio eutéctico, sin tener algún otro agente modificador presente.

- Una buena modificación del silicio eutéctico comienza a presentarse más allá del $1.5 \%$ en peso de sal agregada por gravedad.

- La disociación parcial del $\mathrm{NaCl}$ en el baño metálico limitó la modificación del silicio eutéctico en las muestras tratada con 0.5 y $1.5 \%$ en peso de sal.

- La insuflación del $\mathrm{NaCl}$ a la aleación líquida mediante gas argón desfavoreció el efecto modificador del $\mathrm{Na}$ debido principalmente a que disminuye la presión metalostática de la aleación líquida favoreciendo su evaporación.

\section{AGRADECIMIENTOS}

Agradecemos al COSNET su apoyo para el financiamiento de este trabajo. Al CINVESTAV unidad Saltillo por facilitar sus laboratorios. Al Instituto Tecnológico de Saltillo por facilitar su microscopio electrónico de barrido.

\section{REFERENCIAS}

[1] "Know your Competition... 37th Census of World Casting Production - 2002". Modern Casting Staff Report. Modern Casting, pp. 23-25. Diciembre 2003.

[2] M.M. Haque and M.A. Maleque. "Effect of Process Variables on Structure and Properties of AluminumSilicon Piston Alloy". Journal of Materials Processing Technology. Vol. 77, pp. 122-128. 1998. 
[3] J.W. Zindel and A.K. Dahle. "Eutectic Nucleation and Growth in Hypoeutectic Al-Si Alloys at Different Strontium Levels". Metallurgical and Materials Transactions. Vol. 32A, pp. 949-960. 2001.

[4] John E. Gruzleski and Bernard Closset. "The Treatment of Liquid Aluminum-Silicon Alloys". American Foundrymen's Society. Inc. Des Plaines, Illinois, USA. 1990.

[5] L. Backerud, G. Chai and J. Tamminen. "Solidification Characteristics of Aluminum Alloys". AFS/SKANALUMINIUM. Vol 2, pp. 71-229. 1990.

[6] K. Nogita, A. Knuutinen, S.D. McDonald, A.K. Dahle. "Mechanism of Eutectic Solidification in Al-Si Alloys modified with Ba, Ca, Y and Yd". Journal of Light Metals. Vol. 1, pp. 219-228. 2001.

[7] A. Knuutinen, K. Nogita, S.D. McDonald and A.K. Dahle. "Porosity Formation in Aluminum Alloy A356 Modified with Ba, Ca, Y and Yb". Journal of Light Metals. Elsevier Science. Vol. 1 $\mathrm{N}^{\mathrm{o}}$ 4, pp. 241-249. Noviembre 2001.

[8] A.K. Dahle, K. Nogita, S.D. McDonald, C. Dinnis and L. Lu. "Eutectic Modification and Microstructure Development in Al-Si Alloys". Materials Science and Engineering. Vol. A 413414, pp. 243-248. 2005.

[9] L. Lu, K. Nogita and A.K. Dahle. "Combining Sr and $\mathrm{Na}$ Additions in Hypoeutectic Al-Si Foundry Alloys". Materials Science and Engineering. Vol. A 399, pp. 244-253. 2005.
[10] Enrique J. Martínez D. y R.A. Ramos C. "Estudio del Efecto de la Sal Común en la Porosidad de una Aleación de Aluminio Hipoeutéctica de Bajo Silicio". Memorias del 26 Congreso Internacional de Metalurgia y Materiales. Instituto Tecnológico de Saltillo, pp. 448-449. 2004.

[11] Donna L. Zalensas. "Aluminum Casting Technology”. 2nd edition. AFS, USA, pp. 19-43. 1993.

[12] A.K. Dahle and K. Nogita, J.W. Zinder, S.D. McDonald and L.M. Hogan. "Eutectic Nucleation and Growth in Hypereutectic Al-Si Alloys at Different Strontium Levels". Metallurgical and Materials Transaction. Vol. A 32, pp. 949-960. 2001.

[13] R. Reed - Hill. "Principios de Metalurgia Física". Ed. CECSA. Segunda Edición. 1986.

[14] F.H. Samuel and H.W. Doty. "Factors Controlling the Type and Morphology of Cu-Containing Phases in 319 Al Alloy". AFS Transactions. Vol. 104, pp. 893-901. 1996.

[15] E.J. Martínez D., J. Lacaze, M. Cisneros y S. Valtierra. "Efecto del Estroncio en las Temperaturas de Reacción Eutécticas y Microestructuras de Solidificación de una aleación Al-Si tipo A319. Rev. Fac. Ing. - Univ. Tarapacá. Vol. 12 No 2, pp. 21-26. Chile. 2004.

[16] E. J. Martínez D., M.A. Cisneros G., S. Valtierra and J. Lacaze. "Effect of Strontium and Cooling Rate Upon Eutectic Temperatures of A319 Aluminum Alloy”. Scripta Materialia. Vol. 52, pp. 439-443. 2005. 